\title{
MicroRNA-342-3p IOaded by Human Umbilical Cord Mesenchymal Stem Cells-derived Exosomes Attenuates Deep Vein Thrombosis by Downregulating EDNRA Running Title: Exosome Delivery of miR-342-3p in DVT
}

\author{
Zhiyu Pan \\ Minhang Hospital, Fudan University \\ Qian Chen \\ Minhang Hospital, Fudan University \\ Hongjian Ding \\ Minhang Hospital, Fudan University \\ Huaqing Li ( $\sim$ Lihuaqing166@163.com ) \\ Minhang Hospital, Fudan University
}

\section{Research Article}

Keywords: Deep vein thrombosis, Human umbilical cord mesenchymal stem cells, Exosomes, microRNA342-3p, Endothelin A receptor, Angiogenesis

Posted Date: November 1st, 2021

DOI: https://doi.org/10.21203/rs.3.rs-1023320/v1

License: (c) (i) This work is licensed under a Creative Commons Attribution 4.0 International License. Read Full License 


\section{Abstract}

Objective: Exosomes (exos) exert biological functions to maintain the dynamic balance of cells and tissues by transferring biological cargo to recipient cells. Thus, this study explored human umbilical cord mesenchymal stem cells (hucMSCs)-derived exo transfer of microRNA (miR)-342-3p in deep vein thrombosis (DVT).

Methods: HucMSCs were isolated and transfected with miR-342-3p antagomir/agomir. Then, hucMSCsexos were extracted and injected into rats with DVT to observe inflammation and pathological damage in thrombotic vein. HucMSCs-exos were co-cultured with human umbilical vein endothelial cells (HUVECs) in vitro to observe angiogenesis. miR-342-3p and endothelin A receptor (EDNRA) expression in rats with DVT, as well as their interaction was analyzed.

Results: miR-342-3p was downregulated and EDNRA was upregulated in rats with DVT. HucMSCs-exos induced the repair of venous thrombosis and suppressed inflammation and pathological injury in rats with DVT, as well as promoted angiogenesis of HUVECs. Upregulated miR-342-3p delivery by hucMSCsexos alleviated DVT in rats and improved angiogenesis of HUVECs, while downregulated miR-342-3p performed oppositely. miR-342-3p targeted EDNRA, and the effect of hucMSCs-exos transfer of upregulated miR-342-3p was rescued by overexpressing EDNRA.

Conclusion: Briefly, miR-342-3p loaded by hucMSCs-exos attenuates DVT by downregulating EDNRA, offering a novel direction to treat DVT.

\section{Introduction}

Deep vein thrombosis (DVT) is mostly diagnosed in the legs, deep veins of the arms, visceral veins and cerebral veins [1]. DVT may be asymptomatic, but it usually presents with non-specific symptoms such as discomfort or pain or warmth in the legs, and classically complains swelling, tenderness, pain, or blue/red discoloration in the extremities [2]. Estimation of clinical probability, measurement of D-dimer level and ultrasound are the main diagnostic methods of suspected DVT [3]. Low molecular weight heparin and fondaparinux are standard methods in the initial treatment of patients with DVT, and the duration of anticoagulation therapy should be determined by the balance between the risk of recurrent venous thromboembolism and that of treatment-induced bleeding [4]. However, after stopping anticoagulant drugs, recurrent DVT is usually suspected [5], therefore, development of long-lasting and effective drugs is of importance in DVT.

Human umbilical cord mesenchymal stem cells (hucMSCs) are characterized by faster self-renewal and low immunogenicity, and are concerned as an optimal choice for cell-based therapy [6, 7]. Exosomes (exos) are extracellular vesicles that change the biochemical characteristics of recipient cells through the delivery of biomolecules. The function of MSCs-exos is similar to that of MSCs with low immunogenicity [8]. MSCs-exos could transport miR-145 to protect against atherosclerosis through regulating the migration of human umbilical vein endothelial cells (HUVECs) [9]. Also, H-Y Mao et al. have 
illustrated that MSCs-exos could carry miR-28-3p to pulmonary endothelial cells, causing cellular apoptosis in pulmonary embolism, a major complication of DVT [10]. miR-342-3p has been explored to critically modulate inflammation response and lipid uptake of macrophages (THP-1) in atherosclerosis [11], as well as angiogenesis in vascular endothelial dysfunction [12]. Bioinformatics evaluation identified endothelin A receptor (EDNRA) as a target of miR-342-3p. Olaf Mercier et al. have reported that anti-EDNRA therapy could accelerate the reversal of pulmonary vasculopathy [13]. In fact, downregulating EDNRA at least brings to bear endothelial injury in hypertensive vascular remodeling [14] and can increase vessel sprouting, enhance physiological angiogenesis and decrease pathological neovascularization in ischemic retinopathy [15]. Considering the active functions of miR342-3p and EDNRA in vascular pathology, we performed the research to discover the role of hucMSCs-exo transfer of miR-342-3p in DVT through targeting EDNRA.

\section{Materials And Methods}

\section{Ethics statement}

The animal protocol was reviewed and approved by the Experimental Animal Ethics Committee of Minhang Hospital, Fudan University.

\section{Experimental animals}

Male SD rats $(250 \pm 20 \mathrm{~g})$ were provided by Minhang Hospital and housed with humidity $50-60 \%$ and light/dark cycle $12 / 12 \mathrm{~h}$ at $20-23^{\circ} \mathrm{C}$.

\section{Collection and identification of hucMSCs}

HucMSCs (7530, ScienCell Research Laboratories, CA, USA) were subcultured to passage 4 in the medium (7501, ScienCell Research Laboratories).

The typical surface markers of hucMSCs were tested by flow cytometry. HucMSCs were resuspended in bovine serum albumin and combined with CD29 (1:100, 553715, BD Biosciences, CA, USA), CD34 (1:200, ab81289, Abcam, MA, USA), CD73 (1:50, 550257, BD Biosciences) and CD90 (1:100, 555595, BD Biosciences), respectively. HucMSCs were detected by flow cytometry after resuspending with phosphatebuffered saline (PBS).

HucMSCs were placed in osteogenic medium ( $0.1 \mathrm{mM}$ dexamethasone, $10 \mathrm{mM} \beta$-glycerophosphate and $50 \mathrm{mM}$ ascorbyl phosphate) or adipogenic medium (Cyagen Biosciences, CA, USA). Two weeks later, the osteogenic and adipogenic differentiation potentials were evaluated by Alizarin Red and Oil Red $O$ staining $[16,17]$.

\section{Cell transfection}


miR-342-3p negative control (NC), miR-342-3p agomir and miR-342-3p antagomir (Sangon, Shanghai, China) were utilized to transfect hucMSCs using Lipofectamine 3000 (Invitrogen, CA, USA) [18].

\section{Isolation and identification of hucMSCs-exos}

The supernatant of hucMSCs $(70-80 \%$ confluence) was centrifuged at $300 \times \mathrm{g}, 2000 \times \mathrm{g}$, and $10000 \times \mathrm{g}$ in sequence. The final supernatant was ultracentrifuged at $100000 \times \mathrm{g}$, and then the pellet was then ultracentrifuged at $100000 \times \mathrm{g}$. The pellet was resuspended in PBS, passed through a $0.22 \mu \mathrm{m}$ filter and quantified by bicinchoninic acid method.

The extracted pellet was identified by transmission electron microscope (TEM, JEM-2000FX, JEOL, Japan) to observe morphology, by nanoparticle tracking analysis (NTA, NS300, MIL, Malvern, UK) to measure size and concentration, and by Western blot to analyze the surface markers of exos CD9 (1:1000, ab92762), CD63 (1:1000, ab59479), TSG101 (1:1000, ab125011) and Alix (1:1000, ab117600, all from Abcam) [19].

\section{Construction of DVT model}

SD rats were anesthetized by inhaling $2 \%$ isoflurane and kept in a supine position. A midline abdominal incision was made, and then the inferior vena cava (IVC) was sutured with a single 7-0 suture where it passed through the left renal vein. All side branches were interrupted. Finally, the incision was sutured. All rats were euthanized after $14 \mathrm{~d}$, and the thrombotic IVC was collected.

One hour before IVC suture, rats ( $n=10$ /group) were injected with hucMSCs-exos at $300 \mu \mathrm{g}$ into the femoral vein in situ and related plasmids at $10 \mathrm{nmol}$. The model rats were given the same amount of normal saline, and the normal rats were used as the control. The hucMSCs-exos included untreated hucMSCs-exos, or those transfected with miR-342-3p NC, miR-342-3p agomir and miR-342-3p agomir. The plasmids included overexpression (oe)-NC and oe-EDNRA (Sangon) [20, 21].

\section{Enzyme-linked immunosorbent assay (ELISA)}

The thrombotic IVC was homogenized, and the supernatant was amassed. Tumor necrosis factor- $a$ (TNFa, 70-EK282/3-96), interleukin (IL)-6 (70-EK206/3-96) and IL-1ß (70-EK201B/3-96) kits (Multi Sciences, Hangzhou, China) were used. The absorbance at $450 \mathrm{~nm}$ was read on an Epoch microplate reader (BioTek, VT, USA) [17].

\section{Treatment of HUVECs}

HucMSCs were centrifuged at $100000 \mathrm{~g}$ and labeled with the fluorescent dye CM-Dil (Moleculai Probes, OR, USA). HUVECs (ScienCell Research Laboratories) at 80\% confluence, along with CM-Dil-labeled exos were incubated in Dulbecco's Modified Eagle Medium (DMEM). After that, HUVECs were fixed in $4 \%$ paraformaldehyde, treated with 4',6-diamidino-2-phenylindole (Vector Laboratories, CA, USA) and observed under an inverted fluorescence microscope (IX71-A12FL/PH, Olympus, Japan) [22]. 
After co-culture with exos containing miR-342-3p agomir, HUVECs were transfected with oe-NC or oeEDNRA through Lipofectamine 3000 (Invitrogen), and collected $72 \mathrm{~h}$ later for subsequent detection.

\section{Tube formation assay}

HUVECs were seeded in Matrigel-coated 96-well plates (BD Biosciences) and incubated with exos for 12 h. High glucose DMEM supplemented with $10 \%$ FBS was used as a control. HUVECs were observed under an optical microscope (IX53, Olympus, Tokyo, Japan) and the length of tubes was measured by Image J software $[22,23]$.

\section{Hematoxylin-eosin (H\&E) staining}

An incision was made on the inner thigh skin, the rat thrombus was obtained, and then the femoral vein was separated, and its length and weight were calculated. Thrombotic IVC was fixed with $10 \%$ formaldehyde and embedded in paraffin. The paraffin slices $(4 \mu \mathrm{m})$ were dewaxed with xylene, dehydrated with gradient ethanol, stained with hematoxylin, differentiated with hydrochloric acid and ethanol, and placed in $0.5 \%$ eosin solution. Then, the slices were treated with ethanol for conventional dehydration and xylene for permeabilization, and sealed in neutral resin. The slices were observed under an inverted microscope (XSP-8CA, XTZ Optical Instrument Factory, Shanghai, China) [24, 25].

\section{Masson staining}

The paraffin-embedded thrombotic IVC slices were deparaffinized, stained with Weiger iron-hematoxylin, and differentiated with hydrochloric acid and ethanol. Next, the slices were stained with ponceaunic acid fuchsin solution and treated with $1 \%$ phosphoric acid aqueous solution. Thereafter, the slices were counter-stained with aniline blue solution, treated with $1 \%$ glacial acetic acid, dehydrated with $95 \%$ ethanol and absolute ethanol, permeabilized with xylene, and sealed with neutral resin. The slices were observed under an inverted microscope (XSP-8CA, XTZ Optical Instrument Factory) [26].

\section{Reverse transcription quantitative polymerase chain reaction (RT-qPCR)}

Total RNA was collected from tissues, cells and exos via Trizol (Invitrogen). For miRNA, cDNA was synthesized using TAQMAN MicroRNA Reverse Transcription Kit (Applied Biosystems, CA, USA), and amplified using TAQMAN Micro Assay Mix (Thermo Fisher Scientific); for EDNRA, cDNA was synthesized using a reverse transcription kit (Invitrogen), and analyzed by Sybr Premix EX TAQ II (Takara, Dalian, China). The results were standardized to glyceraldehyde-3-phosphate dehydrogenase (GAPDH) and U6, respectively. The primer sequences are shown in Table 1 . The data was analyzed using the $2^{-\Delta \Delta \mathrm{Ct}}$ method [27].

\section{Western blot assay}

Total protein in tissues, cells and exos was obtained by radio-immunoprecipitation assay lysate containing protease inhibitors, separated by $10 \%$ sodium lauryl sulfate polyacrylamide gel 
electrophoresis, transferred to polyvinylidene fluoride membrane (Millipore, MA, USA) and sealed with 5\% skim milk. The corresponding primary antibodies EDNRA (1:1000, ab85163) and GAPDH (1:1000, ab8245, Abcam), as well as the corresponding secondary antibody were incubated with the membrane. The enhanced chemiluminescence reagent (Beckman Coulter) was used to quantify the protein [26].

\section{Dual luciferase reporter gene assay}

On the bioinformatics website (http://www.targetscan.org), the binding sites of miR-342-3p and EDNRA was predicted. The fluorescein reporter plasmids EDNRA-WT and EDNRA-MUT were produced by QuikChange kit (Agilent Technologies, CA, USA). The constructed reporters were co-transfected with miR342-3p-agomir or miR-342-3p-NC into HEK293T cells, and luciferase activity was assessed by a dual luciferase assay system (Promega, MI, USA) [24].

\section{RNA immunoprecipitation (RIP) assay}

The cell lysate collected by RIPA lysis buffer (P0013B, Beyotime, Shanghai, China). The magnetic beads were resuspended in RIP wash buffer, combined with Ago2 (1:50, ab32381, Abcam) or IgG (1:100, ab109489, Abcam). The magnetic bead-antibody complex was rinsed with RIP wash buffer, incubated with the cell lysate and treated with proteinase K. The extracted RNA was tested by RT-qPCR $[20,26,28]$.

\section{Statistical analysis}

Data assessment was performed in SPSS 21.0 (IBM, NY, USA). Measurement data were expressed by mean \pm standard deviation. The comparison between two groups of measurement data in normal distribution was evaluated by independent sample t test, while that among multiple groups by one-way analysis of variance, followed by Tukey's post-hoc test. Data comparison at different time points was performed by repeated measures analysis of variance, as well as Bonferroni post-hoc test. $P<0.05$ meant statistically significance.

\section{Results}

\section{HucMSCs-exos contain miR-342-3p}

HucMSCs were identified, and Alizarin Red and Oil Red O staining showed that there were many lipid droplets in the cytoplasm of hucMSC, and the cells became alkaline phosphatase positive (Fig. 1A). Flow cytometry reflected that CD29, CD73 and CD90 were all positively expressed while CD34 was negatively expressed on HucMSCs (Fig. 1B). Therefore, it was regarded that most of the cells obtained were hucMSCs.

HucMSCs-exos were separated and identified. TEM observed 80-120 nm sheet-like structure (Fig. 1C). NTA reflected that the size of exos was mostly in the range of 80-140 nm (Fig. 1D). Western blot demonstrated that CD9, TSG101, CD63, and Alix were all positively expressed (Fig. 1E). Given that, the collected extracellular vehicles were hucMSCs-exos. 
Next, after hucMSCs transfected with miR-342-3p agomir, miR-342-3p expression was elevated in both hucMSCs and hucMSCs-exos; on the contrary, transfection with miR-342-3p antagomir caused miR-342-3p expression downregulated in hucMSCs and hucMSCs-exos (Fig. 1F).

\section{HucMSCs-exos induce the repair of venous thrombosis in rats with DVT}

The uptake of hucMSCs-exos by HUVECs was observed, and the images showed that Dil-labeled exos were located in the cytoplasm of HUVECs (Fig. 2A). After co-culture with exos, angiogenesis of HUVECs was enhanced (Fig. 2B).

In rats with DVT, it was seen that the levels of pro-inflammatory factors TNF-a, IL-1 $\beta$ and IL- 6 were increased (Fig. 2C). H\&E staining and Masson staining depicted that the vascular cells of normal rats were arranged regularly, the cells were not degenerated or necrotic, and the thrombus weight to length ratio increased. In model DVT rats, the vascular tissue structure changed, the cells contracted, and the cell membrane permeability increased; the fiber arrangement was disordered and collagen deposited in a large amount (Fig. 2D-F). After treated with hucMSCs-exos, the inflammatory response and the pathological changes of blood vessels in rats with DVT were inhibited.

\section{Upregulated miR-342-3p delivery by hucMSCsexos alleviates DVT in rats}

Thrombotic IVC was collected to measure miR-342-3p expression by RT-qPCR, and the results implied that miR-342-3p was downregulated in rats with DVT (Fig. 3A).

To explore the repair effect of miR-342-3p on the vascular injury of DVT, hucMSCs-exos carrying upregulated miR-342-3p were injected into DVT rats, and successfully enhanced miR-342-3p expression in thrombotic IVC. Upregulated miR-342-3p delivered by hucMSCs-exos enhanced the angiogenesis in vitro (Fig. 3B). Enriched miR-342-3p transported via hucMSCs-exos reduced the levels of proinflammatory factors and thrombus weight/length ratio, and inhibited the pathological changes of blood vessels in vivo (Fig. 3C-G). On the other hand, delivery of lowered miR-342-3p by hucMSCs-exos resulted in the opposite consequences.

\section{miR-342-3p targets EDNRA}

RT-qPCR and Western blot analyzed the elevated EDNRA expression in vascular tissue of rats with DVT (Fig. 4A). Based on the opposite trend of miR-342-3p and EDNRA in rats with DVT, a targeting relation was assumed between miR-342-3p and EDNRA. The binding site did exist between miR-342-3p and EDNRA on the bioinformatics website (Fig. 4B). By luciferase reporter assay, it was found that miR-342-3p agomir diminished the relative luciferase activity of EDNRA-WT (Fig. 4C). Also, through RIP experiment, enriched miR-342-3p and EDNRA were measured in immunoprecipitated Ago2 (Fig. 4D). Thus, EDNRA was confirmed to be targeted by miR-342-3p.

To further verify the targeting relationship, EDNRA expression level that mediated by miR-342-3p was monitored by RT-qPCR and Western blot. The discovery highlighted that in DVT rats injected with exos 
transporting upregulated miR-342-3p, EDNRA expression was reduced; oppositely, exo transfer of downregulated miR-342-3p caused EDNRA expression elevated in DVT rats (Fig. 4E, F).

\section{The effect of hucMSCsexos transfer of upregulated miR-342-3p is rescued by overexpressing EDNRA}

For better determining the mechanism of miR-342-3p targeting EDNRA on DVT, oe-EDNRA was further injected into DVT rats who had been injected with exos carrying upregulated miR-342-3p. Then, it was recognized that oe-EDNRA successfully heightened EDNRA expression (Fig. 5A), induced inflammatory response (Fig. $5 \mathrm{C}$ ), increased thrombus weight/length ratio and worsened the pathological condition in rats with DVT (Figure 5D-F). Meanwhile, transfection with oe-EDNRA in HUVECs reversed the effect of exos carrying upregulated miR-342-3p on angiogenesis in vitro (Fig. 5B).

\section{Discussion}

DVT is an alarming emergency causing high morbidity and mortality. Till now, advances have been attained to understand the pathology and restrain the progression of the disease. Base on the delivery function of hucMSCs-exos, we uncovered the fact that hucMSCs-exos transfer of upregulated miR-342-3p made a relief of DVT in rats through inhibiting EDNRA.

At first, we independently evaluated the function of hucMSCs-exos in rats with DVT, and finally noticed that administration of hucMSCs-exos suppressed inflammatory insult (diminished levels of TNF-a, IL-1 $\beta$ and IL-6) and alleviated vascular injury. Meanwhile, we further performed in vitro assay and noticed that hucMSCs-exos promoted angiogenesis of HUVECs. Similarly, in rats with traumatic brain injury, treatment with MSC-exos could promote endogenous angiogenesis and impair inflammation [29]. In addition, ucMSC-exos could stimulate angiogenesis and induce femoral fracture healing in vivo, and augment tube-forming ability of HUVECs in vitro [30]. Of note, angiogenesis of HUVECs is improved and functional recovery is accelerated in rats with spinal cord injury after administration with miR-126-modified MSCexos [31]. Xiaomei Teng et al. have verified the therapeutic effects of MSCs-exos on inducing tube formation of HUVECs and blood flow recovery, as well as on depressing inflammation response in rats with myocardial infarction [22]. According to a research finding, it is known that MSCs-exos attenuate brain endothelial cell injury via limiting the production of IL-1 $\beta$, IL-6, and TNF- $a$, and apoptosis of endothelial cells [32]. It is worthy noting that treatment with hucMSC-exos is efficacious to enhance the tube-forming ability of HUVECs, so as to accelerate cutaneous wound healing [33]. Generally speaking, hucMSC-exos have therapeutic values in treating diseases via regulation of angiogenesis and inflammation.

Next, we dedicated the performance of miR-342-3p delivered by hucMSCs-exos in DVT, and eventually validated that hucMSCs-exos transfer of restored miR-342-3p marked a great therapeutic effect on relieving venous injury in rats with DVT and on inducing angiogenesis of HUVECs. On the other hand, hucMSCs-exos delivery of silenced miR-342-3p resulted in opposite consequences in vivo and in vitro. In fact, endothelial miR-342-3p is impaired in type 2 diabetes mellitus, and miR-342-3p depletion causes angiogenic dysfunction in the endothelium [12]. miR-342-3p could block endothelial cell inflammation 
while reduction of miR-342-3p in the arterial endothelium unsatisfactorily promotes atherosclerosis [34]. It is informed that miR-342-3p expression is suppressed in atherosclerosis, whilst induction of miR-342-3p restrains inflammation in THP-1 cells [11]. Indeed, miR-342-3p presents proangiogenic properties in cardiovascular disease and its expression is negatively correlated with the levels of IL-6, IL-8 and TNFa [35].

At last, we proved the regulation of EDNRA by miR-342-3p in DVT, and further convinced that overexpressing EDNRA antagonized restored miR-342-3p-mediated effect on rats with DVT and on HUVECs. Actually, EDNRA expression has been examined to increase in blood-outgrowth endothelial cells in the course of systemic capillary leak syndrome [36]. More intriguingly, the increase trend of EDNRA expression has been tested in vascular smooth muscle cells suffered from lipopolysaccharide [37]. Wei Zhou et al. have validated that elevation of miR-1929-3p decreases blood pressure and attenuates endothelial cell injury in part by decreasing EDNRA expression in hypertensive vascular remodeling [14]. It has been implicated that increased EDNRA function is deleterious for cystic fibrosis airway [38]. Exactly, referral of selective EDNRA antagonist (BQ123) may be effectively adopted in pulmonary hypertension [39] and suppression of EDNRA greatly increases vessel sprouting, enhances angiogenesis and decreases pathological neovascularization in ischemic retinopathy [15].

In short, our study analysis obtained a summary that rich miR-342-3p transported by hucMSCs-exos induces the repair of venous injury of rats with DVT and angiogenesis of HUVECs. Although limited to the pre-clinical stage, our research has created a theoretical basis of developing treatment methods for DVT.

\section{Abbreviations}

Deep vein thrombosis (DVT)

Human umbilical cord mesenchymal stem cells (hucMSCs)

Exosomes (exos)

human umbilical vein endothelial cells (HUVECs)

endothelin A receptor (EDNRA)

negative control (NC)

inferior vena cava (IVC)

overexpression (oe)

Enzyme-linked immunosorbent assay (ELISA)

interleukin (IL) 
Dulbecco's Modified Eagle Medium (DMEM)

Hematoxylin-eosin (H\&E)

Reverse transcription quantitative polymerase chain reaction (RT-qPCR)

glyceraldehyde-3-phosphate dehydrogenase (GAPDH)

RNA immunoprecipitation (RIP)

\section{Declarations}

\section{Funding}

None

\section{Ethics approval and consent to participate}

The animal protocol was reviewed and approved by the Experimental Animal Ethics Committee of Minhang Hospital, Fudan University.

\section{Conflict of interest}

The Authors declare no conflicts of interest directly related to the contents of this article.

\section{Consent for publication}

Not applicable

\section{Availability of data and material}

Not applicable

\section{Authors' contributions}

Huaqing Li contributed to study design; Zhiyu Pan contributed to manuscript editing; Qian Chen contributed to experimental studies; Hongjian Ding contributed to data analysis.

All authors read and approved the final manuscript.

\section{Acknowledgement}

We thank the associate editor and the reviewers for their useful feedback that improved this paper.

\section{References}

1. Di Nisio, M., N. van Es, and H.R. Buller, Deep vein thrombosis and pulmonary embolism. Lancet, 2016. 388(10063): p. 3060-3073.

2. Thachil, J., Deep vein thrombosis. Hematology, 2014. 19(5): p. 309-10.

3. Bernardi, E. and G. Camporese, Diagnosis of deep-vein thrombosis. Thromb Res, 2018. 163: p. 201206. 
4. Goldhaber, S.Z. and H. Bounameaux, Pulmonary embolism and deep vein thrombosis. Lancet, 2012. 379(9828): p. 1835-46.

5. Maufus, M., et al., Diagnosis of deep vein thrombosis recurrence: Ultrasound criteria. Thromb Res, 2018. 161: p. 78-83.

6. Ding, D.C., et al., Human umbilical cord mesenchymal stem cells: a new era for stem cell therapy. Cell Transplant, 2015. 24(3): p. 339-47.

7. Li, T., et al., Human umbilical cord mesenchymal stem cells: an overview of their potential in cellbased therapy. Expert Opin Biol Ther, 2015. 15(9): p. 1293-306.

8. Yaghoubi, Y., et al., Human umbilical cord mesenchymal stem cells derived-exosomes in diseases treatment. Life Sci, 2019. 233: p. 116733.

9. Yang, W., et al., Mesenchymal stem-cell-derived exosomal miR-145 inhibits atherosclerosis by targeting JAM-A. Mol Ther Nucleic Acids, 2021. 23: p. 119-131.

10. Mao, H.Y., L.N. Liu, and Y.M. Hu, Mesenchymal stem cells-derived exosomal miRNA-28-3p promotes apoptosis of pulmonary endothelial cells in pulmonary embolism. Eur Rev Med Pharmacol Sci, 2020. 24(20): p. 10619-10631.

11. Wang, L., et al., Blockade of NEAT1 represses inflammation response and lipid uptake via modulating miR-342-3p in human macrophages THP-1 cells. J Cell Physiol, 2019. 234(4): p. 5319-5326.

12. Cheng, S., et al., T2DM inhibition of endothelial miR-342-3p facilitates angiogenic dysfunction via repression of FGF11 signaling. Biochem Biophys Res Commun, 2018. 503(1): p. 71-78.

13. Mercier, O., et al., Endothelin A receptor blockade improves regression of flow-induced pulmonary vasculopathy in piglets. J Thorac Cardiovasc Surg, 2010. 140(3): p. 677-83.

14. Zhou, W., et al., MicroRNA19293p participates in murine cytomegalovirusinduced hypertensive vascular remodeling through Ednra/NLRP3 inflammasome activation. Int J Mol Med, 2021. 47(2): p. 719-731.

15. Patel, C., et al., Activation of the endothelin system mediates pathological angiogenesis during ischemic retinopathy. Am J Pathol, 2014. 184(11): p. 3040-51.

16. Wu, P., et al., HucMSC exosome-delivered 14-3-3zeta alleviates ultraviolet radiation-induced photodamage via SIRT1 pathway modulation. Aging (Albany NY), 2021. 13(8): p. 11542-11563.

17. Dong, B., et al., Exosomes from human umbilical cord mesenchymal stem cells attenuate the inflammation of severe steroid-resistant asthma by reshaping macrophage polarization. Stem Cell Res Ther, 2021. 12(1): p. 204.

18. Du, X., et al., miR-21 induces endothelial progenitor cells proliferation and angiogenesis via targeting FASLG and is a potential prognostic marker in deep venous thrombosis. J Transl Med, 2019. 17(1): p. 270.

19. Cao, J.Y., et al., Exosomal miR-125b-5p deriving from mesenchymal stem cells promotes tubular repair by suppression of p53 in ischemic acute kidney injury. Theranostics, 2021. 11(11): p. 52485266. 
20. Ai, P., et al., MiR-411 suppressed vein wall fibrosis by downregulating MMP-2 via targeting HIF1alpha. J Thromb Thrombolysis, 2018. 45(2): p. 264-273.

21. Sun, J., et al., Endothelial progenitor cell-derived exosomes, loaded with miR-126, promoted deep vein thrombosis resolution and recanalization. Stem Cell Res Ther, 2018. 9(1): p. 223.

22. Teng, X., et al., Mesenchymal Stem Cell-Derived Exosomes Improve the Microenvironment of Infarcted Myocardium Contributing to Angiogenesis and Anti-Inflammation. Cell Physiol Biochem, 2015. 37(6): p. 2415-24.

23. Song, B.W., et al., 1H-pyrrole-2,5-dione-based small molecule-induced generation of mesenchymal stem cell-derived functional endothelial cells that facilitate rapid endothelialization after vascular injury. Stem Cell Res Ther, 2015. 6: p. 174.

24. Ou, M., et al., Upregulated MiR-9-5p Protects Against Inflammatory Response in Rats with Deep Vein Thrombosis via Inhibition of NF-kappaB p50. Inflammation, 2019. 42(6): p. 1925-1938.

25. Tang, K.C., et al., Effect of miR-495 on lower extremity deep vein thrombosis through the TLR4 signaling pathway by regulation of IL1R1. Biosci Rep, 2018. 38(6).

26. Sun, S., et al., Overexpressed microRNA-103a-3p inhibits acute lower-extremity deep venous thrombosis via inhibition of CXCL 12. IUBMB Life, 2020. 72(3): p. 492-504.

27. Du, X., et al., miR-150 regulates endothelial progenitor cell differentiation via Akt and promotes thrombus resolution. Stem Cell Res Ther, 2020. 11(1): p. 354.

28. Zhang, Y., et al., IL (Interleukin)-6 Contributes to Deep Vein Thrombosis and Is Negatively Regulated by miR-338-5p. Arterioscler Thromb Vasc Biol, 2020. 40(2): p. 323-334.

29. Zhang, Y., et al., Effect of exosomes derived from multipluripotent mesenchymal stromal cells on functional recovery and neurovascular plasticity in rats after traumatic brain injury. J Neurosurg, 2015. 122(4): p. 856-67.

30. Zhang, Y., et al., Exosomes from human umbilical cord mesenchymal stem cells enhance fracture healing through HIF-1alpha-mediated promotion of angiogenesis in a rat model of stabilized fracture. Cell Prolif, 2019. 52(2): p. e12570.

31. Huang, J.H., et al., Exosomes Derived from miR-126-modified MSCs Promote Angiogenesis and Neurogenesis and Attenuate Apoptosis after Spinal Cord Injury in Rats. Neuroscience, 2020. 424: p. 133-145.

32. Kong, L.Y., et al., Mesenchymal Stem Cell-derived Exosomes Rescue Oxygen-Glucose Deprivationinduced Injury in Endothelial Cells. Curr Neurovasc Res, 2020. 17(2): p. 155-163.

33. Liu, J., et al., Exosomes Derived from Human Umbilical Cord Mesenchymal Stem Cells Accelerate Cutaneous Wound Healing by Enhancing Angiogenesis through Delivering Angiopoietin-2. Stem Cell Rev Rep, 2021. 17(2): p. 305-317.

34. Jung, Y.Y., et al., Atherosclerosis is exacerbated by chitinase-3-like-1 in amyloid precursor protein transgenic mice. Theranostics, 2018. 8(3): p. 749-766. 
35. Ray, S.L., et al., The Role of miR-342 in Vascular Health. Study in Subclinical Cardiovascular Disease in Mononuclear Cells, Plasma, Inflammatory Cytokines and PANX2. Int J Mol Sci, 2020. 21(19).

36. Sek, A.C., et al., Endothelial Expression of Endothelin Receptor $A$ in the Systemic Capillary Leak Syndrome. PLoS One, 2015. 10(7): p. e0133266.

37. Zhang, W., et al., Enhanced Endothelin A and B Receptor Expression and Receptor-Mediated Vasoconstriction in Rat Mesenteric arteries after Lipopolysaccharide Challenge. Mediators Inflamm, 2019. 2019: p. 6248197.

38. Darrah, R., et al., EDNRA variants associate with smooth muscle mRNA levels, cell proliferation rates, and cystic fibrosis pulmonary disease severity. Physiol Genomics, 2010. 41(1): p. 71-7.

39. Ozen, G., et al., Interaction between PGI2 and ET-1 pathways in vascular smooth muscle from GroupIII pulmonary hypertension patients. Prostaglandins Other Lipid Mediat, 2020. 146: p. 106388.

\section{Tables}

Table 1 Primer sequences

\begin{tabular}{ll} 
Gene & Primers $\left(5^{\prime}\right.$-3 $\left.^{\prime}\right)$ \\
\hline miR-342-3p & F: GGGTCTCACACAGAAATCGC \\
\hline R: CAGTGCGTGTCGTGGAGT \\
\hline EDNRA & F: GCTCTAGATAGGTAGCAACGTGGCTTT \\
\hline R: GCTCTAGAGCCCCAAAACTTGTCAAC \\
\hline F: GGAGCGAGATCTCTCCAAAAT \\
\hline U6 & R: GGCTGTTGTCATACTTCTCATGG \\
\hline & R: AACGCTCTTCGGCAGCACA \\
\hline
\end{tabular}

Note: F, forward; R, reverse; miR-342-3p, microRNA-342-3p; EDNRA, Endothelin A receptor; GAPDH, glyceraldehyde-3-phosphate dehydrogenase.

\section{Figures}


A
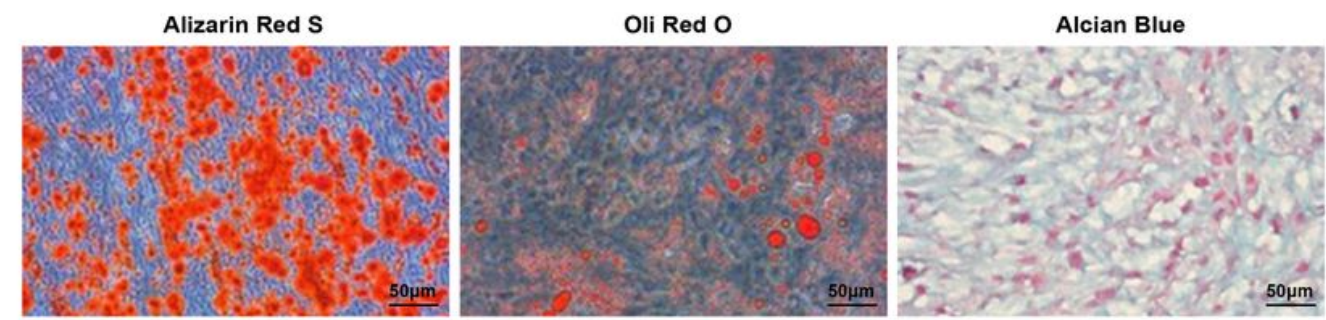

C

B
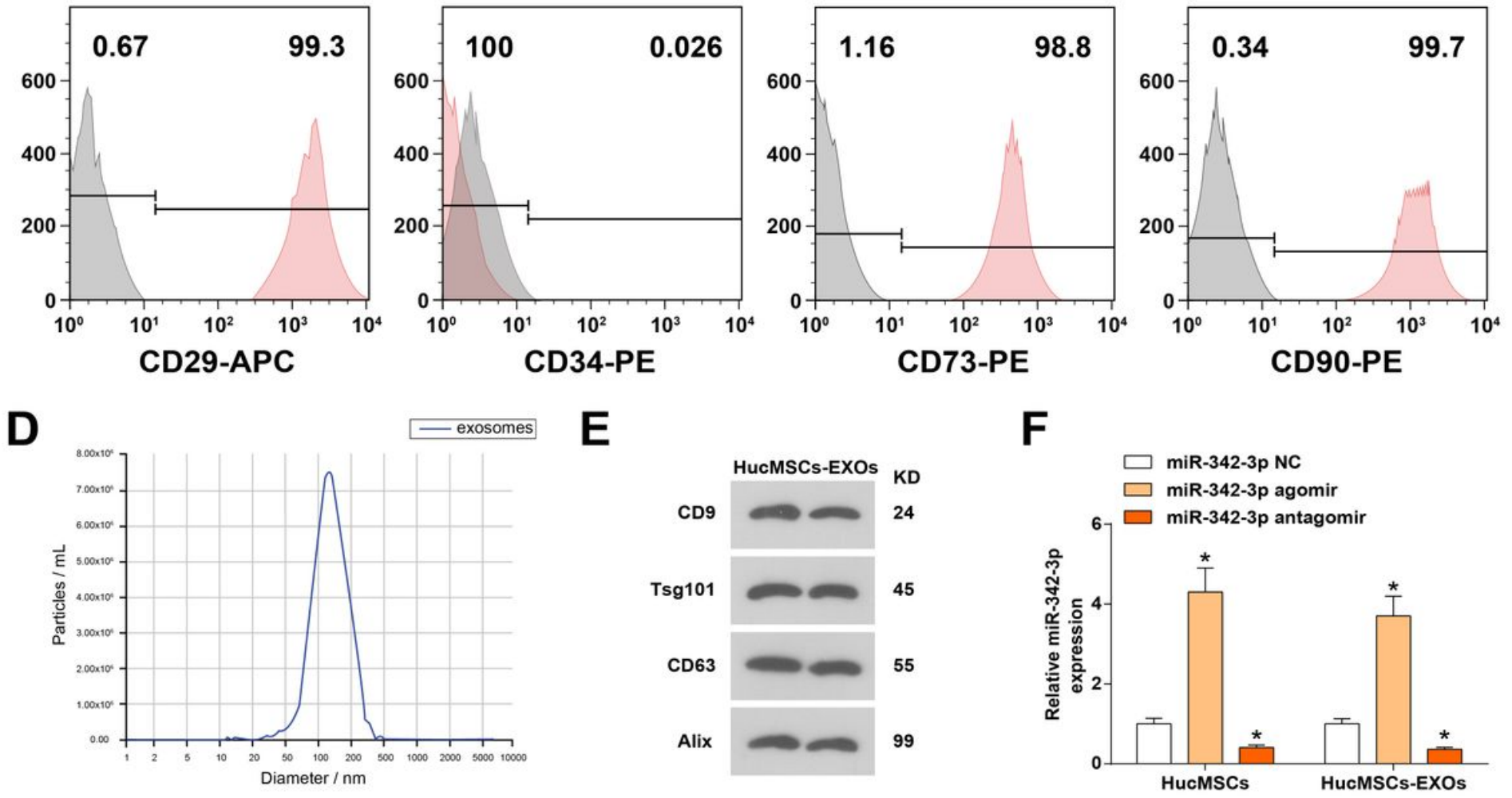

\section{Figure 1}

HucMSCs-exos contain miR-342-3p. A. Alizarin Red staining, Oil Red 0 staining and Alcian Blue staining of hucMSCs (Scale bar: $50 \mu \mathrm{m}$ ); B. Detection of hucMSCs surface antigens by flow cytometry; C.

Identification of exos by TEM; D. Identification of exos by NTA; E. Western blot detection of CD9, TSG101, CD63 and Alix expression; F. miR-342-3p expression levels in hucMSCs and hucMSCs-EXOs;

measurement data were expressed by mean \pm standard deviation; ${ }^{*} P<0.05$ vs. the miR-342-3p NC group. 


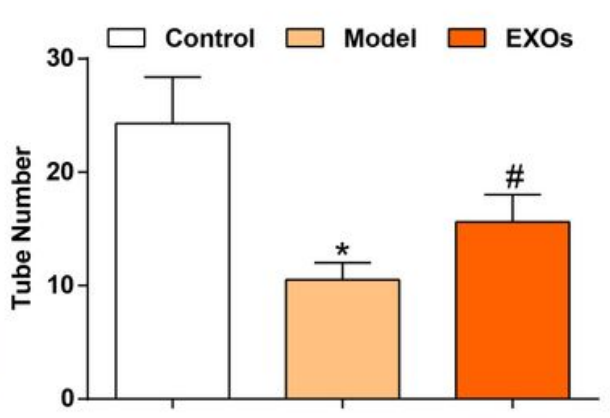

C
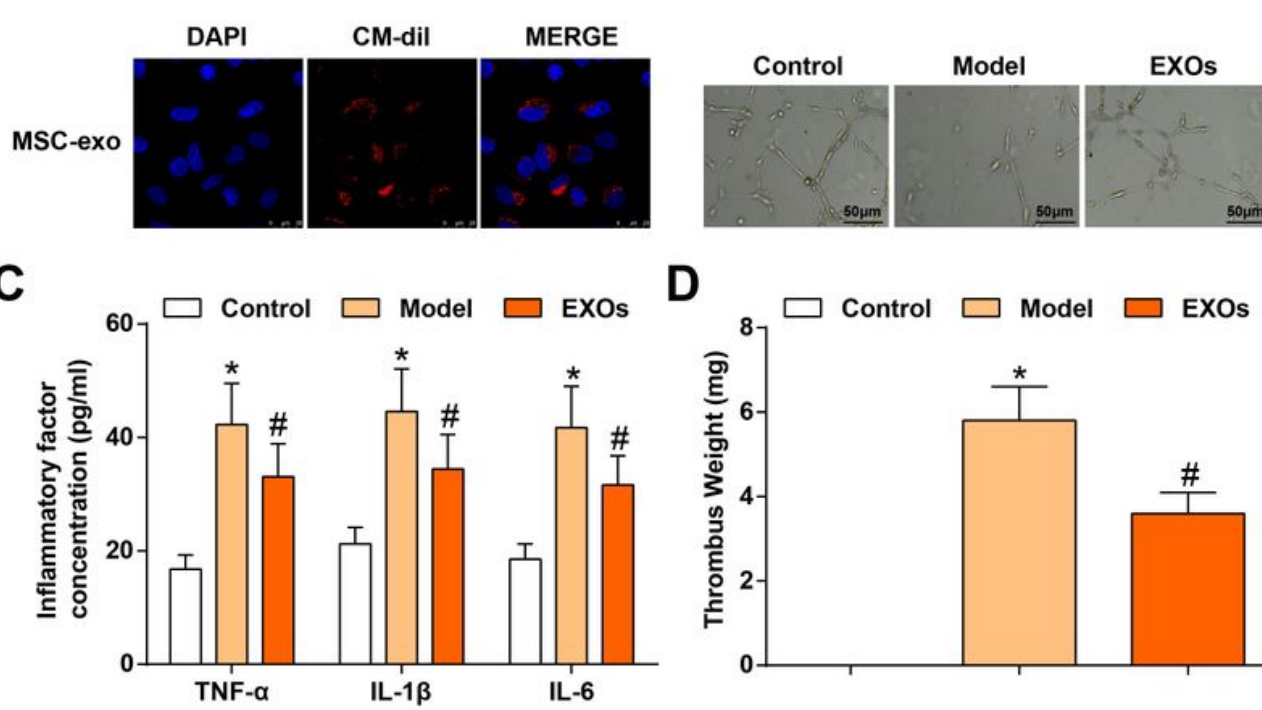

D
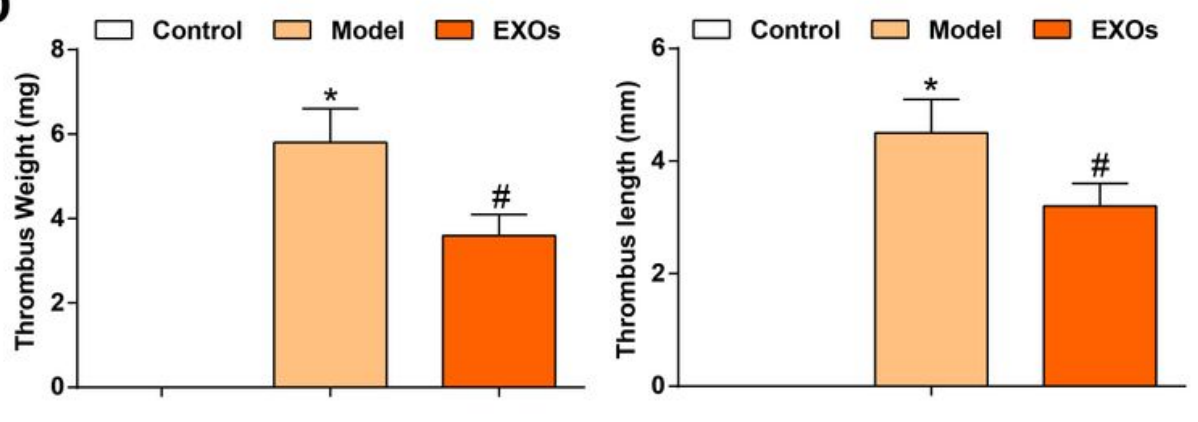

$\mathbf{E}$

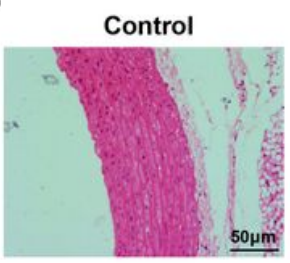

Model EXOs

$\mathbf{F}$
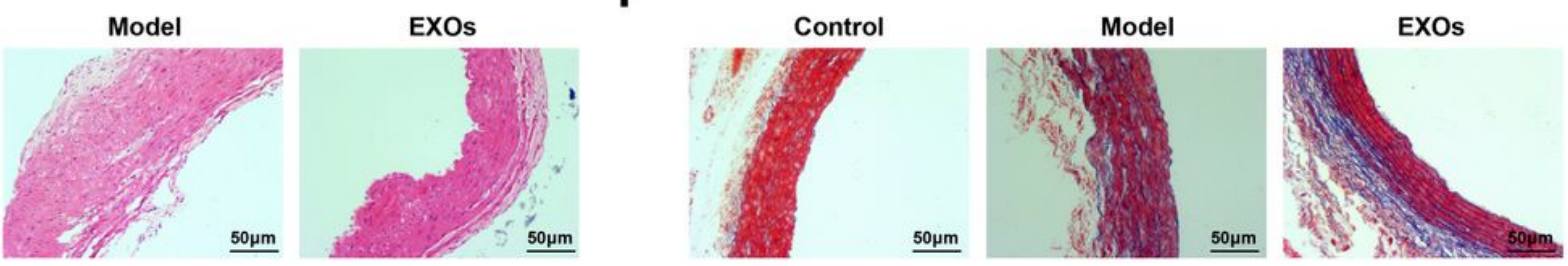

Figure 2

HucMSCs-exos induce the repair of venous thrombosis in rats with DVT. A. Uptake of hucMSCs-exos; B. In vitro angiogenesis; C. TNF- $\alpha$, IL-1 $\beta$, and IL-6 levels in rats after injection with exos; D. Thrombus weight and length; E. HE staining (200 ×); F. Masson staining (200 ×); measurement data were expressed by mean \pm standard deviation; ${ }^{*} \mathrm{P}<0.05$ vs. the Control group; \# $\mathrm{P}<0.05$ vs. the Model group. 
A

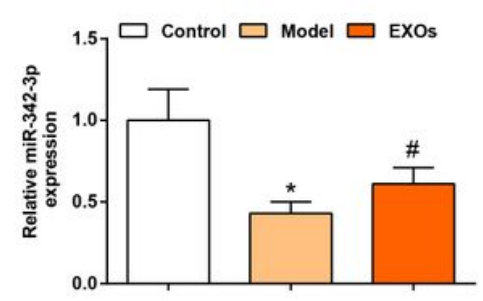

C

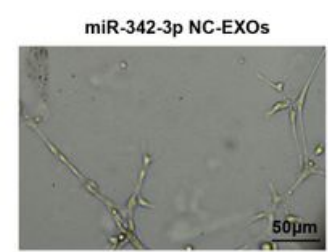

miR-342-3p agomir-EXOs

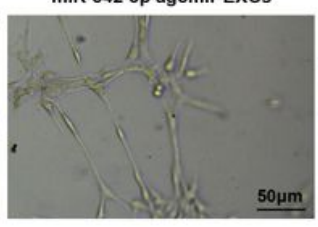

$\mathbf{F}$

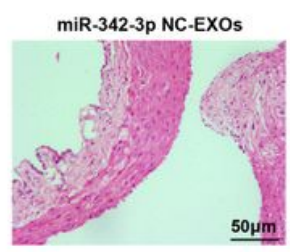

miR-342-3p antagomir-EXOs

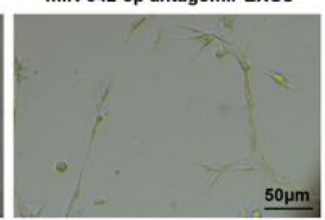

B

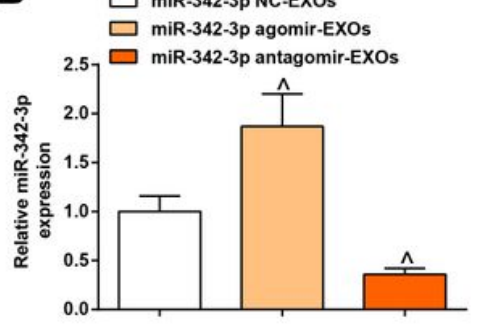

$\underline{50 \mu \mathrm{m}}$

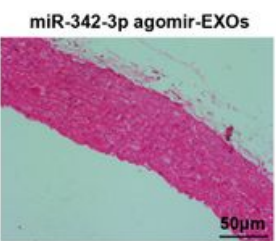

miR-342-3p antagomir-EXOs

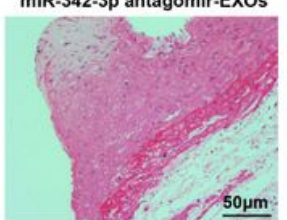

E
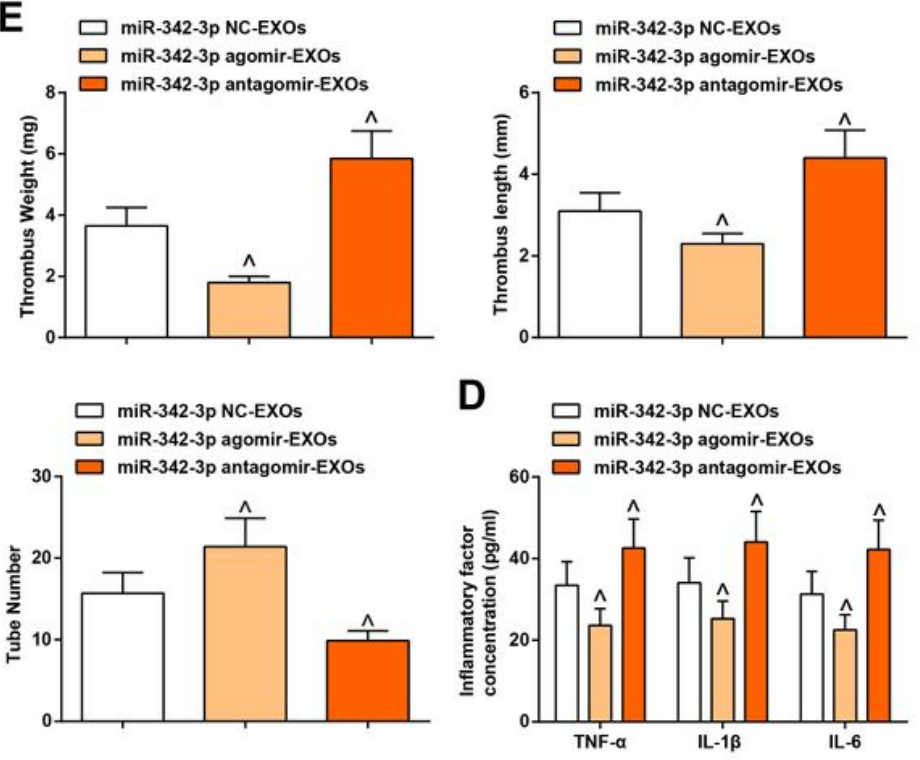

G

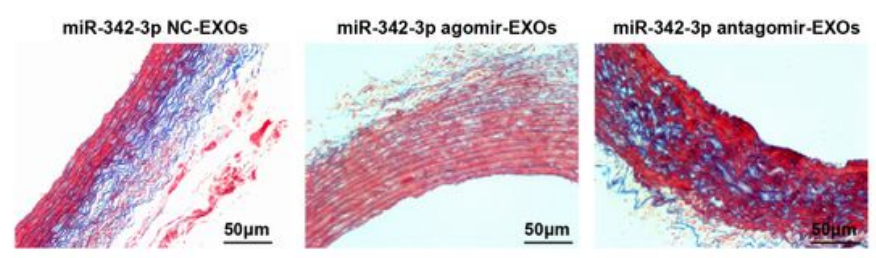

Figure 3

Upregulated miR-342-3p delivery by hucMSCs-exos alleviates DVT in rats. A. miR-342-3p expression level in DVT rats; B. miR-342-3p expression level in DVT rats after injection with upregulated or downregulated exosomal miR-342-3p; C. In vitro angiogenesis; D. TNF- $a$, IL-1 3 , and IL- 6 levels in rats after injection with upregulated or downregulated exosomal miR-342-3p; E. Thrombus weight and length; F. HE staining (200 x); G. Masson staining (200 x); measurement data were expressed by mean \pm standard deviation; * $P<$ 0.05 vs. the Control group; \# P $<0.05$ vs. the Model group; ${ }^{\wedge} \mathrm{P}<0.05$ vs. the miR-342-3p NC-EXOs group. 

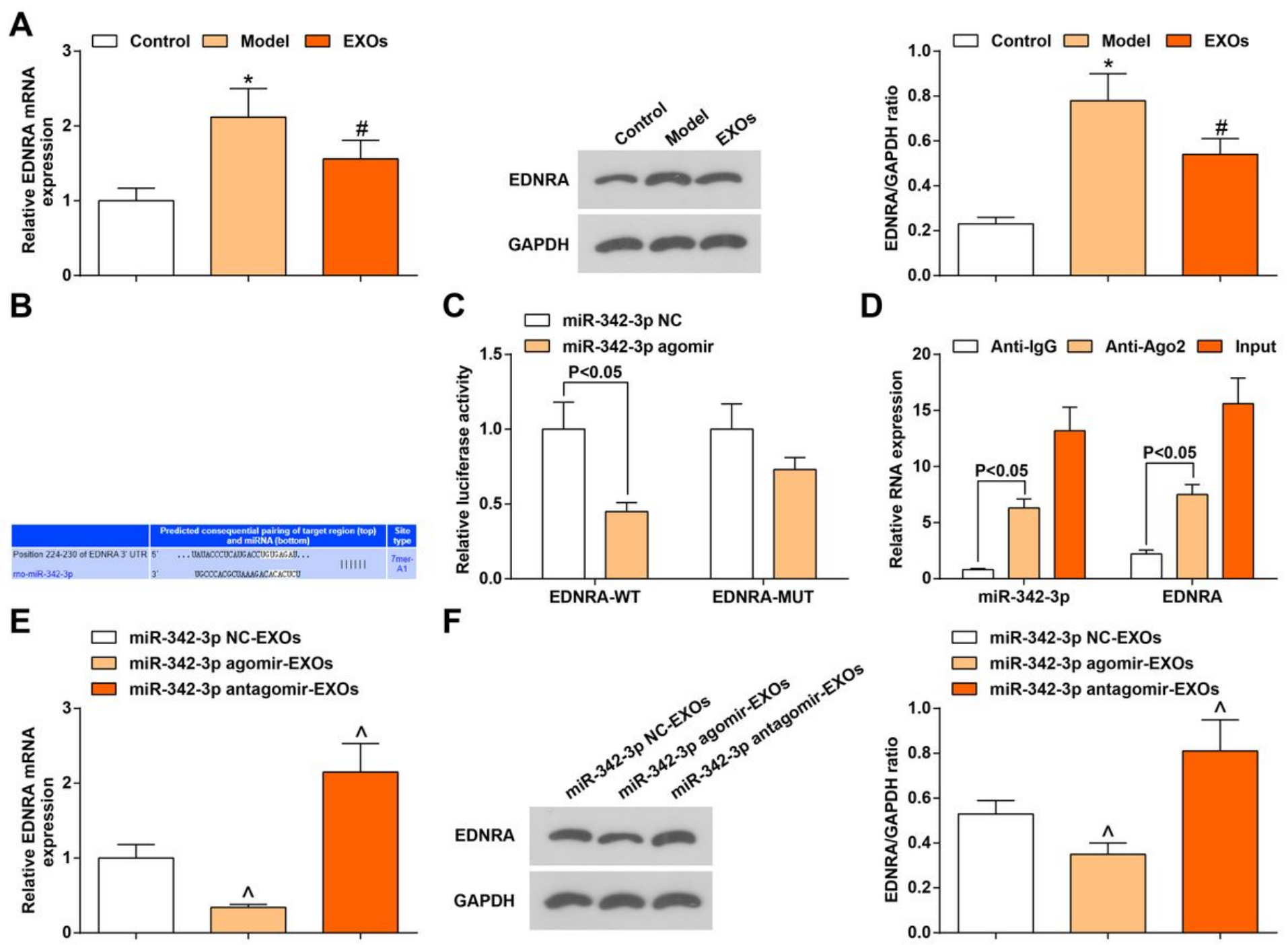

D

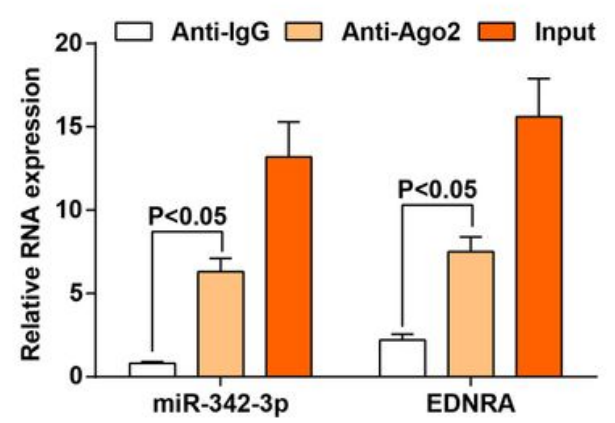

$\mathbf{F}$

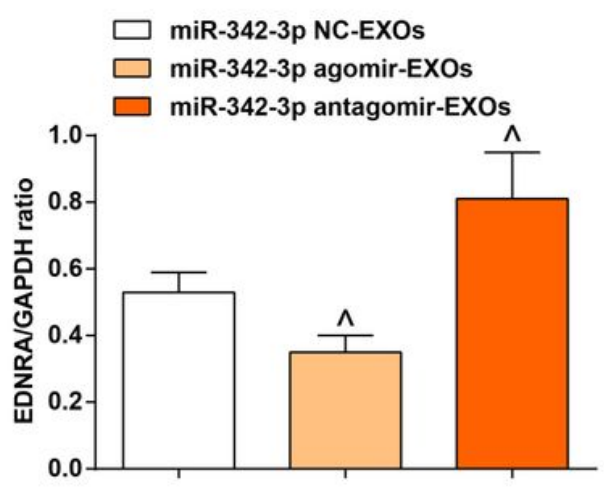

Figure 4

miR-342-3p targets EDNRA. A. EDNRA expression level in DVT rats; $B$. The binding site of miR-342-3p and EDNRA on bioinformatics website; $C$-D. The targeting relationship between miR-342-3p and EDNRA analyzed by dual luciferase reporter gene assay and RIP experiment; E-F. EDNRA expression in rats after regulating exosomal miR-342-3p. Measurement data were expressed by mean \pm standard deviation; * $\mathrm{P}<$ 0.05 vs. the Control group; \# $P<0.05$ vs. the Model group; ${ }^{\wedge} P<0.05$ vs. the miR-342-3p NC-EXOs group. 
A

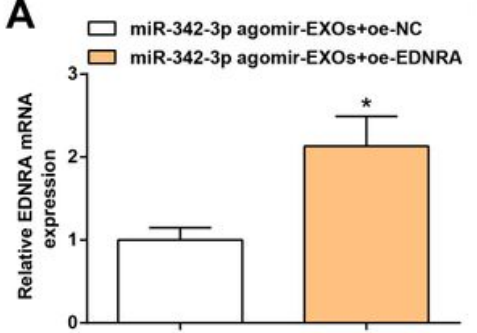

D

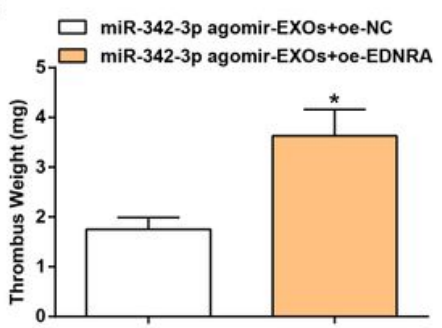

B EXOs+oe-NC EXOs+oe-EDNRA

miR-342-3p agomir-
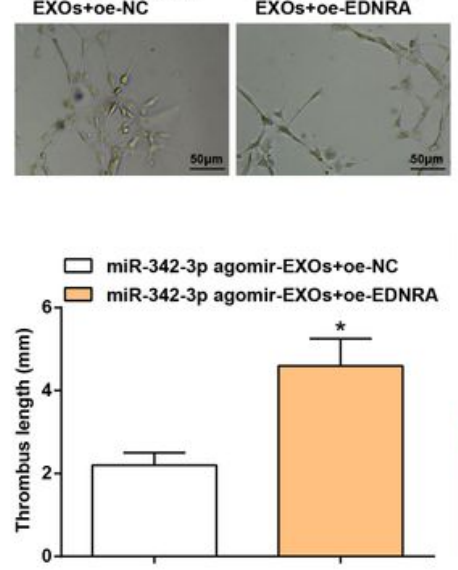

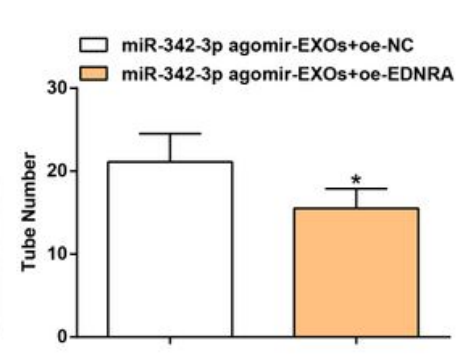

E

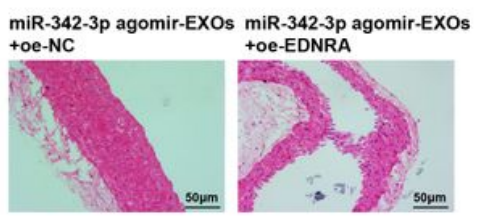

C

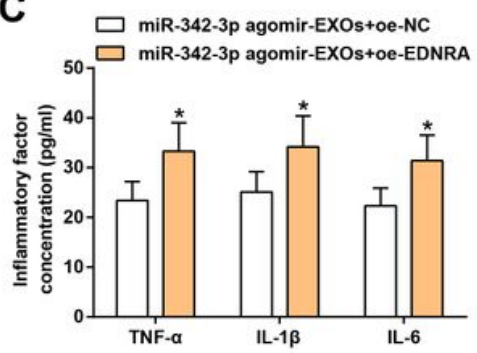

$\mathbf{F}$

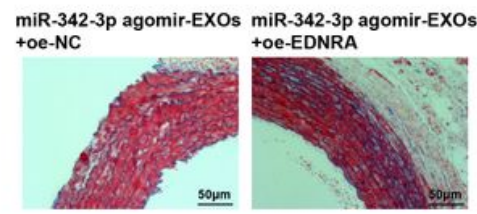

Figure 5

The effect of hucMSCs-exos transfer of upregulated miR-342-3p is rescued by overexpressing EDNRA. A. EDNRA mRNA expression level; B. In vitro angiogenesis; C. TNF-a, IL-1 $\beta$, and IL- 6 expression in rats after overexpressing miR-342-3p and EDNRA; D. Thrombus weight and length; E. HE staining (200 ×); F. Masson staining $(200 \times)$; measurement data were expressed by mean \pm standard deviation; * $P<0.05$ vs. the miR-342-3p agomir-EXOs + oe-NC group. 